\title{
CRP Regulator Modulates Multidrug Resistance of Escherichia coli by Repressing the mdtEF Multidrug Efflux Genes
}

\author{
Kunihiko Nishino, Yasuko Senda, Akihito Yamaguchi
}

Received: September 25, 2007 / Accepted: February 26, 2008

(C) Japan Antibiotics Research Association

\begin{abstract}
Multidrug efflux pumps contribute to the resistance of Escherichia coli against many antibiotics and biocides. Here, we report that the CRP regulator modulates multidrug resistance in E. coli through repression of the genes encoding the MdtEF multidrug efflux pump. Screening of mutants for ability to increase $\beta$-lactam resistance in $E$. coli led to the identification of a mutation in crp, which codes for the major global regulator of catabolite-sensitive operons. Deletion of crp significantly increased the resistance of the $E$. coli strain to oxacillin, azithromycin, erythromycin and crystal violet. The increase in drug resistance caused by $c r p$ deletion was completely suppressed by deletion of the multifunctional outer membrane channel gene tolC. TolC interacts with different drug efflux pumps. Among the twenty drug efflux pumps in E. coli, quantitative real-time PCR analysis showed that CRP repressed the expression of $m d t E F$. Deletion of $m d t E F$ completely suppressed CRP-modulated multidrug resistance. Therefore, in addition to its role in catabolite control, CRP contributes to multidrug resistance in E. coli. Our results indicate that the CRP regulator modulates multidrug resistance in $E$. coli by repressing expression of the MdtEF multidrug efflux pump.
\end{abstract}

Keywords CRP, efflux pump, Escherichia coli, MdtEF, multidrug resistance

K. Nishino, A. Yamaguchi (Corresponding authors), Y. Senda, Department of Cell Membrane Biology, Institute of Scientific and Industrial Research, Osaka University, 8-1 Mihogaoka, Ibaraki, Osaka 567-0047, Japan, E-mail: nishino@sanken.osaka-u.ac.jp; akihito@sanken.osaka-u.ac.jp

K. Nishino: PRESTO, Japan Science and Technology Agency,

\section{Introduction}

Multidrug efflux pumps cause serious problems in cancer chemotherapy and the treatment of bacterial infections. In bacteria, resistance to drugs is associated with multidrug efflux pumps that function to decrease cellular drug accumulation $[1,2]$. Such pumps are classified into the following five families on the basis of sequence similarity: 1) the major facilitator, 2) resistance-nodulation-cell division (RND), 3) small multidrug resistance, 4) multidrug and toxic compound extrusion, and 5) ATP-binding cassette families [3 5]. In Gram-negative bacteria, pumps belonging to the RND family are especially effective in generating resistance $[1,6 \sim 8]$. The sequencing of bacterial genomes enables us to trace putative drug-resistance genes $[9,10]$. There are many putative and proven drug efflux pumps in the genome of Escherichia coli. Our previous studies have shown that $E$. coli has twenty functional drug efflux pumps [11]. Because many of these pumps have overlapping substrate spectra [11], it is intriguing that bacteria, with their economically organized genomes, harbor such large sets of multidrug efflux genes.

The key to understanding how bacteria utilize these multiple pumps lies in the regulation of pump expression. Currently available data show that multidrug efflux pumps are often expressed under precise and elaborate transcriptional control [12 15]. Expression of acrAB,

Tokyo, Japan

A. Yamaguchi: CREST, Japan Science and Technology Agency, Tokyo, Japan

K. Nishino, Y. Senda, A. Yamaguchi: Graduate School of Pharmaceutical Sciences, Osaka University, Osaka, Japan 
Table 1 Escherichia coli strains used in this study

\begin{tabular}{|c|c|c|c|}
\hline Strain or Plasmid & Original name & Characteristics & Source or references \\
\hline \multicolumn{4}{|l|}{ Strains as in text } \\
\hline KAM3 & KAM3 & Derivative of TG1 that lacks $a c r B$ & 21 \\
\hline$\Delta c r p$ & NKE 1220 & $\mathrm{KAM} 3 \Delta c r p$ & This study \\
\hline$\Delta$ tolC & NKE 822 & $\mathrm{KAM} 3 \Delta$ tolC & This study \\
\hline$\Delta$ tolC $\Delta c r p$ & NKE 1221 & $\mathrm{KAM} 3 \Delta$ tolC $\Delta \mathrm{crp}$ & This study \\
\hline$\Delta m d t E F$ & NKE 356 & $\mathrm{KAM} 3 \Delta m d t E F$ & 34 \\
\hline$\Delta m d t E F \Delta c r p$ & NKE 1222 & $\mathrm{KAM} 3 \Delta m d t E F \Delta c r p$ & This study \\
\hline
\end{tabular}

which encodes the major AcrAB efflux pump, is subject to multiple levels of regulation. In $E$. coli, it is modulated locally by the local repressor AcrR [16]. At a more global level, it is modulated by stress conditions and by global regulators such as MarA, SoxS and Rob [17, 18]. These examples illustrate the complexity and diversity of the mechanisms regulating bacterial multidrug efflux pumps.

The E. coli cyclic AMP receptor protein (CRP) is an important transcription factor that regulates the initiation of transcription for more than 100 genes, mainly involved in the catabolism of carbon sources other than glucose [19]. $E$. coli utilizes glucose preferentially over other sugars and only catabolizes other sugars when the supply of glucose is depleted [20]. The presence of glucose prevents E. coli from catabolizing alternative sugars by several mechanisms, one of which is to lower the level of cAMP, the inducer for CRP.

In this study, we show that CRP contributes to multidrug resistance in $E$. coli in addition to its role in catabolite control. The results suggest a previously uncharacterized physiological role for CRP in multidrug resistance.

\section{Materials and Methods}

\section{Bacterial Strains, Plasmids and Growth Conditions}

The bacterial strains and plasmids used in this study are listed in Table 1 . The E. coli strains used are derived from the strain KAM3 [21]. Bacterial strains were grown at $37^{\circ} \mathrm{C}$ in Luria-Bertani (LB) broth [22]. Cells were rapidly collected for total RNA extraction when the cultures reached an optical density of 0.6 at $600 \mathrm{~nm}$.

\section{Screening for Regulators of Multidrug Resistance}

DNA manipulation generally followed standard practice [22]. Strain KAM3 was subjected to transposon mutagenesis using the EZ-Tn5 $<$ R6K $\gamma$ ori/KAN-2 $>$ Tnp Transposome kit (Epicenter) according to the manufacturer's instructions. Briefly, the Transposome complex was electroporated into KAM3, then approximately 10,000 colonies were screened. Cells were plated on LB-agar medium [22] containing $25 \mu \mathrm{g} / \mathrm{ml}$ kanamycin and inhibitory concentrations of various drugs. Genomic DNA was prepared from the drug-resistant strains digested with SmaI, then immediately ligated. Ligation products were electroporated into strain EC100D pir-116 and kanamycin-resistant transformants were selected. Plasmid DNAs were purified from these strains and were sequenced with primers KAN-2 FP-1 and R6KAN-2 RP-1 (Epicenter). Sequence data were used to interrogate the $E$. coli MG1655 sequence (http://genolist.pasteur.fr/Colibri/) to identify the sites of transposon insertion.

\section{Construction of Gene Deletion Mutants}

To construct gene deletion mutants from E. coli KAM3 cells [21], precise in-frame deletions were generated by crossover PCR with the primers listed in Table 2, described previously [23]. The fragment containing the deletion was cloned into the BamHI site of the pKO3 vector [23], a gene replacement vector that contains a temperature-sensitive origin of replication and markers for positive and negative selection for chromosome integration and excision. The deletion was introduced into the chromosome by the $\mathrm{pKO} 3$ gene replacement protocol, as described previously [23].

\section{Determination of the MIC for Toxic Compounds}

The antibacterial activities of different agents were determined on L-agar (1.0\% tryptone, $0.5 \%$ yeast extract, $0.5 \% \mathrm{NaCl}$ ) plates containing the compounds (Sigma) indicated in Table 3 at various concentrations. Agar plates were made by the two-fold agar dilution technique as described [24]. To determine the MICs, bacteria were grown in Luria-Bertani broth at $37^{\circ} \mathrm{C}$ overnight and diluted in the same medium, then tested at a final inoculum size of $10^{4} \mathrm{cfu} \mu \mathrm{1}^{-1}$ using a multipoint inoculator (Sakuma Seisakusyo, Tokyo, Japan), after incubation at $37^{\circ} \mathrm{C}$ for 20 hours. The MIC was the lowest concentration of compound that inhibited cell growth. 
Table 2 Primers used in this study

\begin{tabular}{|c|c|}
\hline Primer & Sequence $\left(5^{\prime}\right.$ to $\left.3^{\prime}\right)$ \\
\hline \multicolumn{2}{|c|}{ For gene deletion } \\
\hline crp-No & CGCGCGGCCGCACAATCGACCACATCCTGACGCCC \\
\hline crp-Ni & CACGCAATAACCTTCACACTCCAAATTTATAACCATGCGCGGTTATCCTCTGTTATAAGC \\
\hline crp-Ci & GTTATAAATTTGGAGTGTGAAGGTTATTGCGTGTAATCCCGTCGGAGTGGCGCGTTAC \\
\hline crp-Co & CGCGTCGACCCAGTTAAACAATCCGTACCAGAG \\
\hline tolC-No & CGCGGATCCTCATCCCGGCAACCATCTC \\
\hline tolC-Ni & CACGCAATAACCTTCACACTCCAAATTTATAACCATTCCTTGTGGTGAAGCAGTAT \\
\hline tolC-Ci & GTTATAAATTTGGAGTGTGAAGGTTATTGCGTGTGATGACGACGACGGGG \\
\hline tolC-Co & CGCGGATCCGCTGGATTGCTGGGCC \\
\hline mdtE-No & CGCGGATCCCAGTTCAAAATTATGCAACTGATTCTG \\
\hline mdtE-Ni & CACGCAATAACCTTCACACTCCAAATTTATAACCATTTTAGTCCCTGAAAATTCTTGAG \\
\hline mdtF-Ci & GTTATAAATTTGGAGTGTGAAGGTTATTGCGTGTAACGTGTAAATGAGAGTAAGGTTGA \\
\hline mdtF-Co & CGCGGATCCCGTCAAATTCCTCTGCATACTATTGC \\
\hline \multicolumn{2}{|c|}{ For quantitative PCR } \\
\hline rrsA-F & CGGTGGAGCATGTGGTTTAA \\
\hline $\operatorname{rrs} A-\mathrm{R}$ & GAAAACTTCCGTGGATGTCAAGA \\
\hline acrA-F & GTCTATCACCCTACGCGCTATCTT \\
\hline acrA-R & GCGCGCACGAACATACC \\
\hline acrD-F & GTACCCTGGCGATTTTTTCATT \\
\hline acrD-R & CGGTCACTCGCACATTCG \\
\hline acrE-F & CGTGATTGCCGCAAAAGC \\
\hline acrE-R & TTGGCGCAGTGACTTTGGTA \\
\hline$b c r-F$ & TGTTTTTCTGTTCGTGATGACCAT \\
\hline$b c r-\mathrm{R}$ & GGAACATATTTAACGCGCCAAT \\
\hline cusB-F & CGCTTACCGTGGGCGATA \\
\hline cusB-R & TTCCACCCAGTCAGGAATGG \\
\hline emrA-F & GCGAATATTGAGGTGCAGAAAA \\
\hline emrA-R & GGCACACGGCGGTTGTA \\
\hline emrD-F & GTGGATCCCCGACTGGTTT \\
\hline emrD-R & CCCGGCACCGAAAAAGA \\
\hline emrE-F & GGTATTGTCCTGATTAGCTTACTGTCAT \\
\hline emrE-R & GCACAAATCAACATCATGCCTATAA \\
\hline emrK-F & GCGCTTAAACGTACGGATATTAAGA \\
\hline emrK-R & ACTGTTTCGCCGACCTGAAC \\
\hline$f_{S r}-\mathrm{F}$ & TGGTGTTGGCGCAAATCA \\
\hline$f_{S r}-\mathrm{R}$ & TCGTCGCTTTGGGTTTTCC \\
\hline macA-F & CGGTGATTGCCGCACAA \\
\hline macA-R & TTACCAGCATGGCGCTCAT \\
\hline$m d f A-F$ & CTTGCTGTTAGCGCGTCTGA \\
\hline$m d f A-R$ & GCCAGCCGCCCATAATAAT \\
\hline$m d t A-F$ & CGCCGTAGAACAGGCAGTTC \\
\hline$m d t A-R$ & TGCGCACCGTAACGGTATTA \\
\hline$m d t E-F$ & CCCCCGGTTCGGTCAA \\
\hline$m d t E-R$ & GGACGTATCTCGGCAACTTCAT \\
\hline$m d t F-F$ & TTACCGTCAGCGCTACCTATCC \\
\hline$m d t F-R$ & GCCATCAAGCCCATTCATATTT \\
\hline$m d t G-F$ & CGGTATTGTCTTCAGCATTACATTTT \\
\hline$m d t G-R$ & GGCGAGTCCACCCCAAA \\
\hline$m d t H-F$ & TTTTCACCCTGATTTGTCTGTTTTAT \\
\hline$m d t H-\mathrm{R}$ & CAGCGAAGCACTTAAGGTTTCA \\
\hline$m d t J-F$ & TGATGAAAATTGCCGGGTTAA \\
\hline
\end{tabular}


Table 2 Continued

\begin{tabular}{|c|c|}
\hline$m d t J-R$ & CGCTTTACGGGTACCTGATTTTA \\
\hline$m d t K-R$ & GAAACCTTGTCGCACCTGATG \\
\hline$m d t L-F$ & TATCCCGCCGGGATTGATAT \\
\hline$m d t M-F$ & CGTGATTTTAATGCCGATGTCA \\
\hline$m d t M-R$ & GCCATACCGCCAGCAAGAT \\
\hline tolC-F & CCGGGATTTCTGACACCTCTT \\
\hline tolC-R & TTTGTTCTGGCCCATATTGCT \\
\hline
\end{tabular}

\section{RNA Extraction}

Total RNA was isolated from bacterial cultures using an RNeasy Protect Bacteria Mini Kit (Qiagen) and RNaseFree DNase (Qiagen) as described previously [25]. The absence of genomic DNA from DNase-treated RNA samples was confirmed by both non-denaturing agarose electrophoresis gels and PCR with primers known to target genomic DNA. The RNA concentration was determined spectrophotometrically [22].

\section{Determination of Specific Transcript Levels by Quantitative Real-time PCR Following Reverse Transcription}

Bulk cDNA samples were synthesized from total RNA using TaqMan Reverse Transcription Reagents (PE Applied Biosystems) and random hexamers as described previously $[26,27]$. The specific primer pairs listed in Table 2 were designed using ABI PRISM Primer Express software (PE Applied Biosystems). rrs of the 16S rRNA gene was chosen as the normalizing gene [28]. Real-time PCR was performed with each specific primer pair using SYBR Green PCR Master Mix (PE Applied Biosystems). The reactions were run on an ABI PRISM 7000 Sequence Detection System (PE Applied Biosystems); the fluorescence signal due to SYBR Green intercalation was monitored to quantify the double-stranded DNA product formed in each PCR cycle.

\section{Results}

\section{Mutation in the $\operatorname{crp}$ Locus Increases Resistance to Oxacillin}

Expression of multidrug efflux genes is often regulated in a complex manner, as described in the introduction. We therefore screened the mutants of $E$. coli that increased multidrug resistance levels in this organism. We used a host
Table 3 Susceptibility of $E$. coli strains to $\beta$-lactams and toxic compounds

\begin{tabular}{lcccc}
\hline \multirow{2}{*}{ Strain } & \multicolumn{4}{c}{ MIC $(\mu \mathrm{g} / \mathrm{ml})$} \\
\cline { 2 - 5 } & OXA & AZM & ERM & CV \\
\hline KAM3 & 0.5 & 0.5 & 2 & 0.5 \\
$\Delta$ crp & $\mathbf{4}$ & $\mathbf{4}$ & $\mathbf{1 6}$ & $\mathbf{8}$ \\
$\Delta$ tolC & 0.5 & 0.5 & 2 & 0.5 \\
$\Delta$ tolC $\Delta$ crp & 0.5 & 0.5 & 2 & 0.5 \\
$\Delta$ mdtEF & 0.5 & 0.5 & 2 & 0.5 \\
$\Delta$ mdtEF $\Delta$ crp & 0.5 & 0.5 & 2 & 0.5 \\
\hline
\end{tabular}

OXA, oxacillin; AZM, azithromycin; ERM, erythromycin; CV, crystal violet.

Values in boldface are larger than those of KAM3 strain.

MIC determinations were repeated at least three times.

strain lacking a functional $a c r B$ gene in the screening in order to identify regulatory elements involved in the expression of other multidrug resistance systems. The transposon insertion mutants were made from the strain KAM3 as described in Materials and Methods. The mutants were plated on LB agar containing $25 \mu \mathrm{g} / \mathrm{ml}$ kanamycin and inhibitory concentrations of various drugs. In one experiment, the medium contained $2.0 \mu \mathrm{g} / \mathrm{ml}$ of oxacillin, which had an MIC of $0.5 \mu \mathrm{g} / \mathrm{ml}$ against KAM3. When one of the mutant colonies that grew on this medium was purified and reexamined, we indeed found a 8 -fold increase in oxacillin MIC against the transposon insertion mutant (data not shown).

Sequencing determined that the transposon was inserted into the coding sequence of crp. CRP is the major global regulator of catabolite-sensitive operons and it controls its own synthesis [19]. It seemed possible that deletion of $\mathrm{crp}$ might be causing the transcriptional activation of genes involved in oxacillin resistance. 
To test whether deletion of $c r p$ confers oxacillin resistance on the E. coli KAM3 strain, full-length wild-type crp was deleted as described in Materials and Methods. Oxacillin MICs for cells lacking crp were 8 times higher (4 versus $0.5 \mu \mathrm{g} / \mathrm{ml}$ ) than for KAM3 cells (Table 3), suggesting that the deletion of the CRP regulator indeed conferred oxacillin resistance on E. coli.

\section{Deletion of CRP Increases Resistance of $E$. coli to Multiple Drugs}

Our results had shown that deletion of crp increased E. coli resistance to oxacillin. We therefore investigated the effect of $c r p$ deletion on the susceptibility of $E$. coli to other toxic compounds. Various drugs were tested, including common substrates of multidrug efflux pumps, and we found that crp deletion increased the resistance of the KAM3 strain to azithromycin, erythromycin and crystal violet (Table 3). These results indicate that the deletion of the CRP regulator induces multidrug resistance in E. coli.

\section{Effect of tolC Deletion on the Multidrug Resistance Modulated by CRP}

The results described above indicate that the expression of a multidrug efflux pump may be induced by deletion of $\mathrm{crp}$. In our previous study, it was revealed that at least twenty intrinsic drug efflux transporters are encoded in the $E$. coli chromosome [11]. Among these, RND-family transporters play major roles in both intrinsic and elevated resistance of Gram-negative bacteria to a wide range of noxious compounds including s-lactams [1, 11, 29 31]. RND transporters need two other proteins for their function: a membrane fusion protein and an outer membrane channel. It has been reported that many drug transporter systems in $E$. coli need the membrane channel TolC in order to function [31 34].

To determine whether CRP-mediated multidrug resistance is attributable to the TolC-dependent drug efflux pump(s), we investigated the effect of tolC deletion on the drug resistance of the crp-deleted cells. The tolC deletion completely inhibited CRP-mediated multidrug resistance (Table 3). This result indicates that CRP-mediated multidrug resistance is attributable to increased functioning of a TolC-dependent drug efflux pump.

\section{Determination of the Amounts of Drug Exporter Transcripts by Quantitative Real-time Reverse Transcription-PCR (qRT-PCR)}

In order to determine which drug efflux pump show increased expression when crp is deleted, we used qRTPCR to investigate changes in the amounts of drug efflux gene mRNAs dependent on crp deletion. Total RNAs were isolated from exponential-phase cultures of KAM3 and $\Delta c r p$, and cDNA samples were synthesized using TaqMan reverse transcription reagents (PE Applied Biosysems) with random hexamers as primers. Real-time PCR of the cDNAs was performed with each specific primer pair using SYBR Green PCR Master Mix (PE Applied Biosystems). The expression levels of drug efflux pump genes and tolC in $\Delta c r p$ were compared with those in KAM3. The results are shown in Table 4. Expression of $m d t E$ was significantly increased. Deletion of $c r p$ did not increase the expression levels of other drug efflux genes and tolC (Table 4).

\section{Effects of Deletion of the MdtEF Drug Efflux Pump on CRP-modulated Multidrug Resistance}

In order to determine whether multidrug resistance mediated by crp deletion is due to increased expression of $m d t E F$, we investigated the effects of deleting these genes on drug resistance levels in KAM3 and $\Delta$ crp (Table 3). When $m d t E F$ was deleted from the KAM3 strain there was no change in drug resistance in the resulting strains. In the

Table 4 Fold induction of specific transcripts attributed to crp deletion as determined by qRT-PCR

\begin{tabular}{|c|c|}
\hline Gene & Fold increase \\
\hline acrA & $0.92 \pm 0.067$ \\
\hline$a c r D$ & $1.1 \pm 0.081$ \\
\hline $\operatorname{acr} E$ & $0.95 \pm 0.035$ \\
\hline$b c r$ & $1.2 \pm 0.12$ \\
\hline cusB & $1.1 \pm 0.055$ \\
\hline emrA & $0.99 \pm 0.072$ \\
\hline emrD & $0.96 \pm 0.022$ \\
\hline emrE & $1.1 \pm 0.071$ \\
\hline emrK & $1.6 \pm 0.38$ \\
\hline$f s r$ & $0.83 \pm 0.13$ \\
\hline macA & $0.94 \pm 0.048$ \\
\hline$m d f A$ & $1.1 \pm 0.15$ \\
\hline$m d t A$ & $1.2 \pm 0.26$ \\
\hline$m d t E$ & $\mathbf{3 4} \pm 3.5$ \\
\hline$m d t G$ & $1.1 \pm 0.087$ \\
\hline$m d t H$ & $1.3 \pm 0.28$ \\
\hline mdtJ & $1.2 \pm 0.15$ \\
\hline$m d t K$ & $0.92 \pm 0.086$ \\
\hline$m d t L$ & $0.82 \pm 0.058$ \\
\hline$m d t M$ & $0.85 \pm 0.11$ \\
\hline tolc & $1.2 \pm 0.15$ \\
\hline
\end{tabular}

The amount of transcript was determined by quantitative real-time PCR as described in Materials and Methods. The fold change ratio was calculated by dividing the expression level of the gene in the $\Delta$ crp strain by that in the KAM3 strain. Experiments were performed in triplicate and the data are represented mean values \pm standard deviation. The values in boldface type indicate inreases of more than tenfold. 
$\triangle m d t E F$ strain, deletion of crp conferred no drug resistance (Table 3). Together, these data indicate that the multidrug resistance conferred by deletion of the CRP regulator is due to derepression of the $m d t E F$ multidrug efflux genes.

\section{Discussion}

In this study, we performed a genome-wide search for a regulator of multidrug resistance in E. coli by random insertion and discovered that CRP down-regulates the expression of $m d t E F$. We initially found by random insertion that the mutation in crp conferred oxacillin resistance on the KAM3 strain. Then we investigated the susceptibility of the KAM3 strain lacking crp to various drugs including common substrates of multidrug efflux pumps, and found that CRP modulates $E$. coli resistance to oxacillin, azithromycin, erythromycin and crystal violet (Table 3).

A dominant mechanism by which $E$. coli and other related bacteria sense carbon sufficiency involves cyclic AMP and its receptor protein, CRP [19, 35]. CRP induces a sharp bend in the DNA and is capable of regulating the expression of more than 100 genes. The mechanisms by which CRP regulates gene expression in response to variable cytoplasmic levels of cyclic AMP have been extensively investigated with particular emphasis on $E$. coli and Salmonella strains [35 38]. Dozens of operons have been shown by classical approaches to be subject to CRPmediated control [39]. We have previously reported that $N$ acetyl-D-glucosamine induces the expression of $m d t E F$ and this induction is dependent on $n a g E$ and $c r p$, and inhibited by the addition of cyclic AMP [40]. In this study, we discovered the importance of CRP as a repressor of drug resistance through the regulation of the multidrug efflux pump MdtEF. These data indicates the connection between the control of the multidrug efflux pump and sugar metabolism. In this study, we used a complex growth medium in the absence of glucose. We recently measured the cellular amount of cAMP of the KAM3 strain in a complex growth medium with or without glucose. And we found that glucose significantly reduces the cAMP amount of this strain and enhances its tolerance to multiple drugs (Nishino K. et al., unpublished data).

In addition to the roles of CRP in catabolite control, we found that it contributes to multidrug resistance in E. coli by regulating the MdtEF multidrug efflux pump (Fig. 1). Our results suggest a previously uncharacterized physiological role for CRP in multidrug resistance. Further investigation of the regulation of multidrug efflux systems in several natural environments, such as inside hosts, is

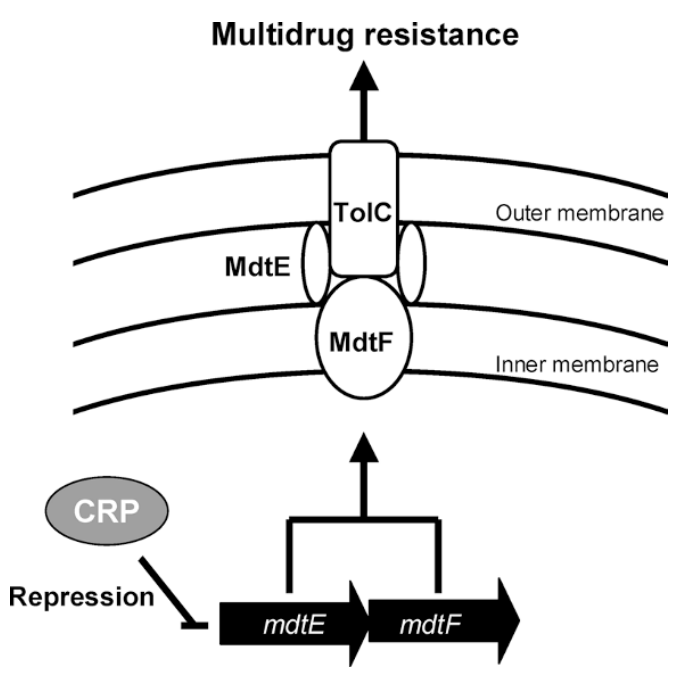

Fig. 1 Model for CRP control of multidrug resistance.

CRP controls expression of mdtEF multidrug efflux pump genes. The evidences in this study shows that CRP represses mdtEF and deletion of crp causes multidrug resistance of Escherichia coli.

needed in order to elucidate the biological significance of their regulatory networks. Such investigation may provide further insights into the role of multidrug efflux systems in the physiology of the cell.

Acknowledgements We thank Geroge $M$. Church and Tomofusa Tsuchiya for providing strains and plasmids; and members of our laboratory for comments on this work. We would also like to thank the anonymous reviewers for their thoughtful comments on this work. This research was supported by a Research Aid from the Inoue Foundation for Science, the Okawa Foundation for Information and Telecommunications, Japan Research Foundation for Clinical Pharmacology, Kato Memorial Bioscience Foundation, Ohyama Health Foundation Inc., Novartis Foundation (Japan) for the Promotion of Science, Takeda Science Foundation, Japan Antibiotics Research Association, Daiwa Securities Health Foundation, Yakult Bio-Science Foundation, the Zoonosis Control Project of the Ministry of Agriculture, Forestry and Fisheries of Japan, a Grant-in-Aid for Young Scientists (S) from the Japan Society for the Promotion of Science, PRESTO, Japan Science and Technology Agency, Japan (to K.N.), the Program for Promotion of Fundamental Studies in Health Sciences of the National Institute of Biomedical Innovation, and a Grant from the Ministry of Education, Culture, Sports, Science and Technology of Japan (to K.N. and A.Y.).

\section{References}

1. Nikaido H. Multidrug efflux pumps of Gram-negative bacteria. J Bacteriol 178: 5853-5859 (1996) 
2. Zgurskaya HI, Nikaido H. Multidrug resistance mechanisms: drug efflux across two membranes. Mol Microbiol 37: 219-225 (2000)

3. Putman M, van Veen HW, Konings WN. Molecular properties of bacterial multidrug transporters. Microbiol Mol Biol Rev 64: 672-693 (2000)

4. Brown MH, Paulsen IT, Skurray RA. The multidrug efflux protein NorM is a prototype of a new family of transporters. Mol Microbiol 31: 394-395 (1999)

5. Paulsen IT, Chen J, Nelson KE, Saier MH. Comparative genomics of microbial drug efflux systems. J Mol Microbiol Biotechnol 3: 145-150 (2001)

6. Murakami S, Nakashima R, Yamashita E, Yamaguchi A. Crystal structure of bacterial multidrug efflux transporter AcrB. Nature 419: 587-593 (2002)

7. Yu EW, Aires JR, Nikaido H. AcrB multidrug efflux pump of Escherichia coli: composite substrate-binding cavity of exceptional flexibility generates its extremely wide substrate specificity. J Bacteriol 185: 5657-5664 (2003)

8. Murakami S, Nakashima R, Yamashita E, Matsumoto T, Yamaguchi A. Crystal structures of a multidrug transporter reveal a functionally rotating mechanism. Nature 443: 173-179 (2006)

9. Paulsen IT, Sliwinski MK, Saier MH. Microbial genome analyses: global comparisons of transport capabilities based on phylogenies, bioenergetics and substrate specificities. J Mol Biol 277: 573-592 (1998)

10. Paulsen IT, Nguyen L, Sliwinski MK, Rabus R, Saier MH. Microbial genome analyses: comparative transport capabilities in eighteen prokaryotes. J Mol Biol 301: 75-100 (2000)

11. Nishino K, Yamaguchi A. Analysis of a complete library of putative drug transporter genes in Escherichia coli. J Bacteriol 183: 5803-5812 (2001)

12. Ahmed M, Borsch CM, Taylor SS, Vazquez-Laslop N, Neyfakh AA. A protein that activates expression of a multidrug efflux transporter upon binding the transporter substrates. J Biol Chem 269: 28506-28513 (1994)

13. Brooun A, Tomashek JJ, Lewis K. Purification and ligand binding of EmrR, a regulator of a multidrug transporter. J Bacteriol 181: 5131-5133 (1999)

14. Lomovskaya $\mathrm{O}$, Lewis $\mathrm{K}$, Matin A. EmrR is a negative regulator of the Escherichia coli multidrug resistance pump
EmrAB. J Bacteriol 177: 2328-2334 (1995)

15. Grkovic S, Brown MH, Skurray RA. Regulation of bacterial drug export systems. Microbiol Mol Biol Rev 66: 671-701 (2002)

16. Ma D, Alberti M, Lynch C, Nikaido H, Hearst JE. The local repressor AcrR plays a modulating role in the regulation of acrAB genes of Escherichia coli by global stress signals. Mol Microbiol 19: 101-112 (1996)

17. Randall LP, Woodward MJ. The multiple antibiotic resistance (mar) locus and its significance. Res Vet Sci 72: 87-93 (2002)

18. Rosenberg EY, Bertenthal D, Nilles ML, Bertrand KP, Nikaido $\mathrm{H}$. Bile salts and fatty acids induce the expression of Escherichia coli AcrAB multidrug efflux pump through their interaction with Rob regulatory protein. Mol Microbiol 48: 1609-1619 (2003)

19. Kolb A, Busby S, Buc H, Garges S, Adhya S. Transcriptional regulation by cAMP and its receptor protein. Annu Rev Biochem 62: 749-795 (1993)

20. Postma P. Catabolite repression and related phenomena Symp Soc Gen Microbiol 39: 21-49 (1986)

21. Morita Y, Kodama K, Shiota S, Mine T, Kataoka A, Mizushima T, Tsuchiya T. NorM, a putative multidrug efflux protein, of Vibrio parahaemolyticus and its homolog in Escherichia coli. Antimicrob Agents Chemother 42: 1778-1782 (1998)

22. Sambrook J, Fritsch EF, Maniatis T. Molecular cloning: a laboratory manual, 2nd ed. Cold Spring Harbor Laboratory, Cold Spring Harbor, N.Y. (1989)

23. Link AJ, Phillips D, Church GM. Methods for generating precise deletions and insertions in the genome of wild-type Escherichia coli: application to open reading frame characterization. J Bacteriol 179: 6228-6237 (1997)

24. Nishino K, Yamaguchi A. Role of histone-like protein H-NS in multidrug resistance of Escherichia coli. J Bacteriol 186 : 1423-1429 (2004)

25. Nishino K, Honda T, Yamaguchi A. Genome-wide analyses of Escherichia coli gene expression responsive to the BaeSR two-component regulatory system. J Bacteriol 187: 1763-1772 (2005)

26. Nishino K, Yamaguchi A. Overexpression of the response regulator $\operatorname{evg} A$ of the two-component signal transduction system modulates multidrug resistance conferred by 
multidrug resistance transporters. J Bacteriol 183: 1455-1458 (2001)

27. Nishino K, Inazumi Y, Yamaguchi A. Global analysis of genes regulated by EvgA of the two-component regulatory system in Escherichia coli. J Bacteriol 185: 2667-2672 (2003)

28. Nishino K, Latifi T, Groisman EA. Virulence and drug resistance roles of multidrug efflux systems of Salmonella enterica serovar Typhimurium. Mol Microbiol 59: 126-141 (2006)

29. Ma D, Cook DN, Hearst JE, Nikaido H. Efflux pumps and drug resistance in Gram-negative bacteria. Trends Microbiol. 2: 489-493 (1994)

30. Nikaido H. Prevention of drug access to bacterial targets: permeability barriers and active efflux. Science 264: 382-388 (1994)

31. Nishino K, Yamada J, Hirakawa H, Hirata T, Yamaguchi A. Roles of TolC-dependent multidrug transporters of Escherichia coli in resistance to $\beta$-lactams. Antimicrob Agents Chemother 47: 3030-3033 (2003)

32. Elkins CA, Nikaido H. Substrate specificity of the RNDtype multidrug efflux pumps AcrB and AcrD of Escherichia coli is determined predominantly by two large periplasmic loops. J Bacteriol 184: 6490-6498 (2002)

33. Fralick JA. Evidence that TolC is required for functioning of the Mar/AcrAB efflux pump of Escherichia coli. J Bacteriol 178: 5803-5805 (1996)

34. Nishino K, Yamaguchi A. EvgA of the two-component signal transduction system modulates production of the yhiUV multidrug transporter in Escherichia coli. J Bacteriol 184: 2319-2323 (2002)

35. Saier MH, Ramseier TM, Reizer J. Regulation of carbon utilization, pp. 1325-1343. In Neidhardt FC, Curtiss R, Ingraham JL, Lin ECC, Low KB, Magasanik B, Reznikoff WS, Riley M, Schaechter M, Umbarger HE (ed.), Escherichia coli and Salmonella: cellular and molecular biology, 2nd ed. ASM Press, Washington, D.C. (1996)

36. Busby S, Ebright RH. Transcription activation by catabolite activator protein (CAP). J Mol Biol 293: 199-213 (1999)

37. Saier MH, Chauvaux S, Deutscher J, Reizer J, Ye JJ. Protein phosphorylation and the regulation of carbon metabolism: comparisons in Gram-negative versus gram-positive bacteria. Trends Biochem Sci 20: 267-271 (1995)

38. $\mathrm{Xu}$ J, Johnson RC. Cyclic AMP receptor protein functions as a repressor of the osmotically inducible promoter proP P1 in Escherichia coli. J Bacteriol 179: 2410-2417 (1997)

39. Magasanik B, Neidhardt FC. Regulation of carbon and nitrogen utilization, pp. 1318-1325. In Neidhardt FC, Ingraham JL, Low KB, Magasanik B, Schaechter M, Umbarger HE (ed.), Escherichia coli and Salmonella typhimurium: cellular and molecular biology. American Society for Microbiology, Washington, D.C. (1987)

40. Hirakawa H, Inazumi Y, Senda Y, Kobayashi A, Hirata T, Nishino K, Yamaguchi A. N-Acetyl-D-glucosamine induces the expression of multidrug exporter genes, $m d t E F$, via catabolite activation in Eschrichia coli. J Bacteriol 188: 5851-5858. 